\title{
Delta variant is in decline in the US, Texas, and Lubbock County
}

\author{
Gilbert Berdine MD
}

The last month has brought good news about the surge in COVID cases, hospitalization, and deaths seen in the United States, Texas, and Lubbock County. This surge started around July 1, 2021. The current outbreak has made clear that COVID will be a recurring problem, just like influenza, and will require better preparation to avoid problems during the next outbreak.

Figure 1 illustrates the history of new cases attributed to COVID-19 since the beginning of the pandemic in the United States. The surge due to the Delta variant began around July 1, 2021, and daily new cases peaked on September 2, 2021. The 7-day moving average is clearly in decline.

Figure 2 illustrates daily deaths attributed to COVID-19 in the United States. The surge due to

the Delta variant began around July 10, 2021, and the daily deaths peaked on September 18, 2021. A time lag between the daily cases and daily deaths is expected and is due to average time for the illness to progress from first onset of symptoms to an outcome of death in fatal cases.

Figure 3 illustrates that the course of the Delta variant was similar in Texas compared to the United States as a whole. The surge due to Delta variant began around July 1, 2021, and daily new cases peaked on September 14, 2021. Although there were some reversals in the trend around the time of peak cases, the final decline of the Delta outbreak has clearly begun.

Figure 4 illustrates Texas hospital beds occupied by patients with a diagnosis of COVID-19. The surge

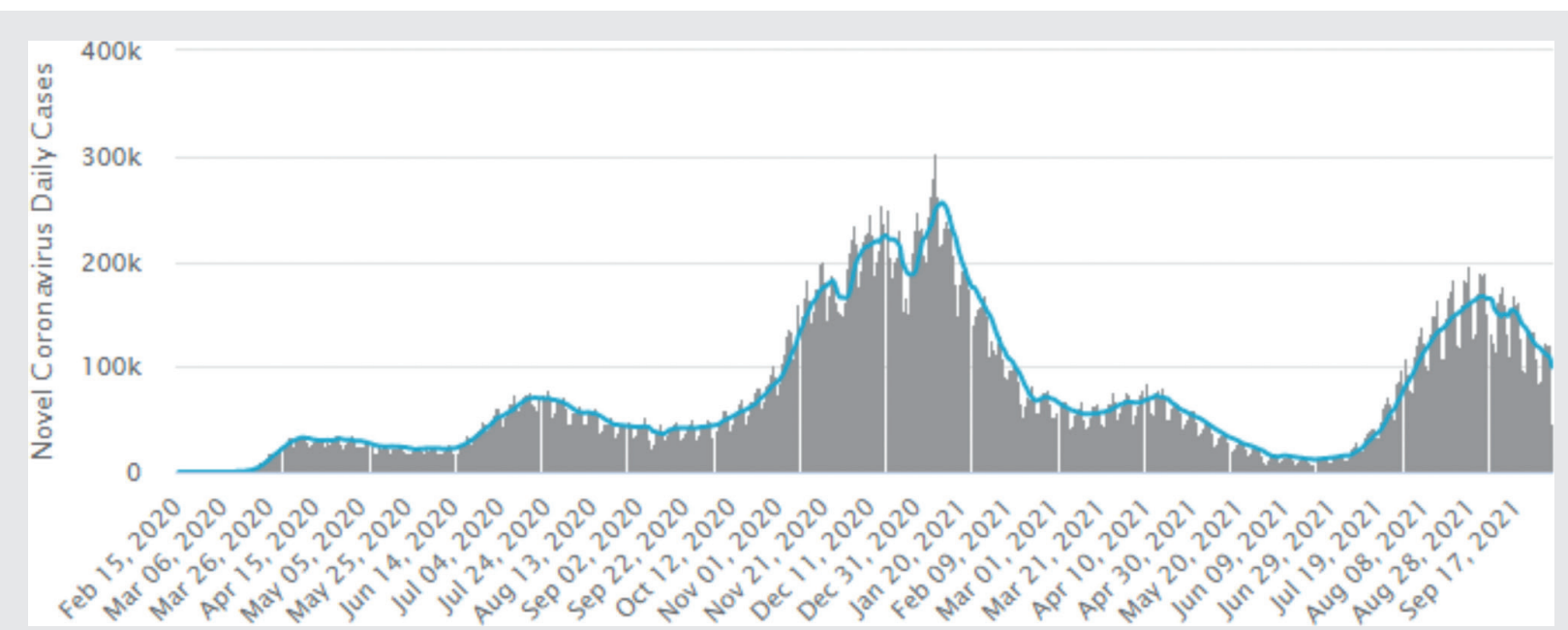

Figure 1. Daily new cases of COVID-19 in the United States. Data are from Worldometer. ${ }^{1}$ The blue curve is the 7-day moving average that smooths out fluctuations due to weekly reporting from individual locations.

Corresponding author: Gilbert Berdine

Contact Information: Gilbert.Berdine@ttuhsc.edu

DOI: $10.12746 /$ swrccc.v9i41.943 


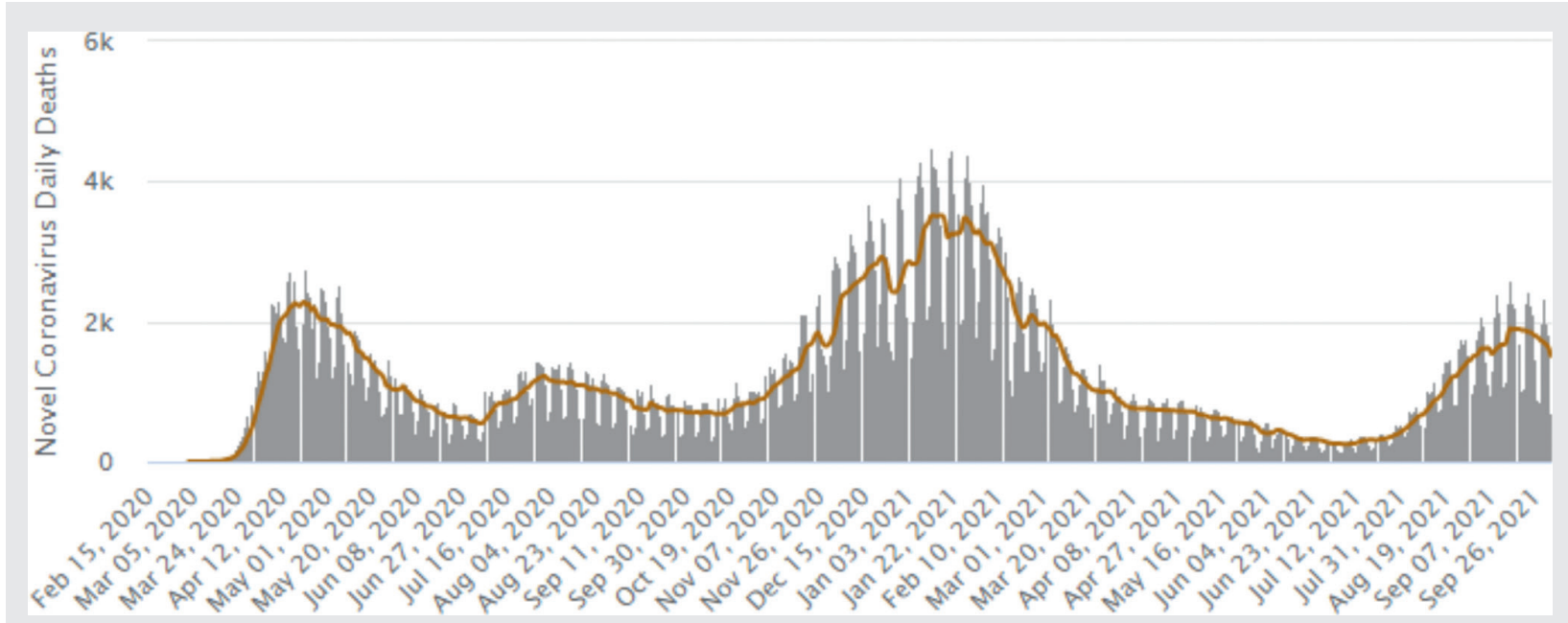

Figure 2. Daily new deaths attributed to COVID-19 in the United States. Data are from Worldometer. ${ }^{1}$ The orange curve is the 7 -day moving average.

due to the Delta variant began around July 1, 2021, and hospitalizations peaked on August 25, 2021, with peak hospitalizations of 13,932 . The hospitalizations peaked before the new cases peaked. The likely explanation is that students arriving at Texas schools from out of state will likely produce a surge of minor cases with few of these cases leading to hospitalization. The fluid situation at the Texas border with
Mexico may also be a factor. Like new cases, Texas hospitalizations from COVID-19 are clearly in decline.

Figure 5 illustrates the daily deaths attributed to COVID-19 in the state of Texas. The surge due to the Delta variant began on July 1, 2021, and daily deaths peaked on September 1, 2021, with peak deaths around 300 per day. The daily deaths are also clearly in decline.

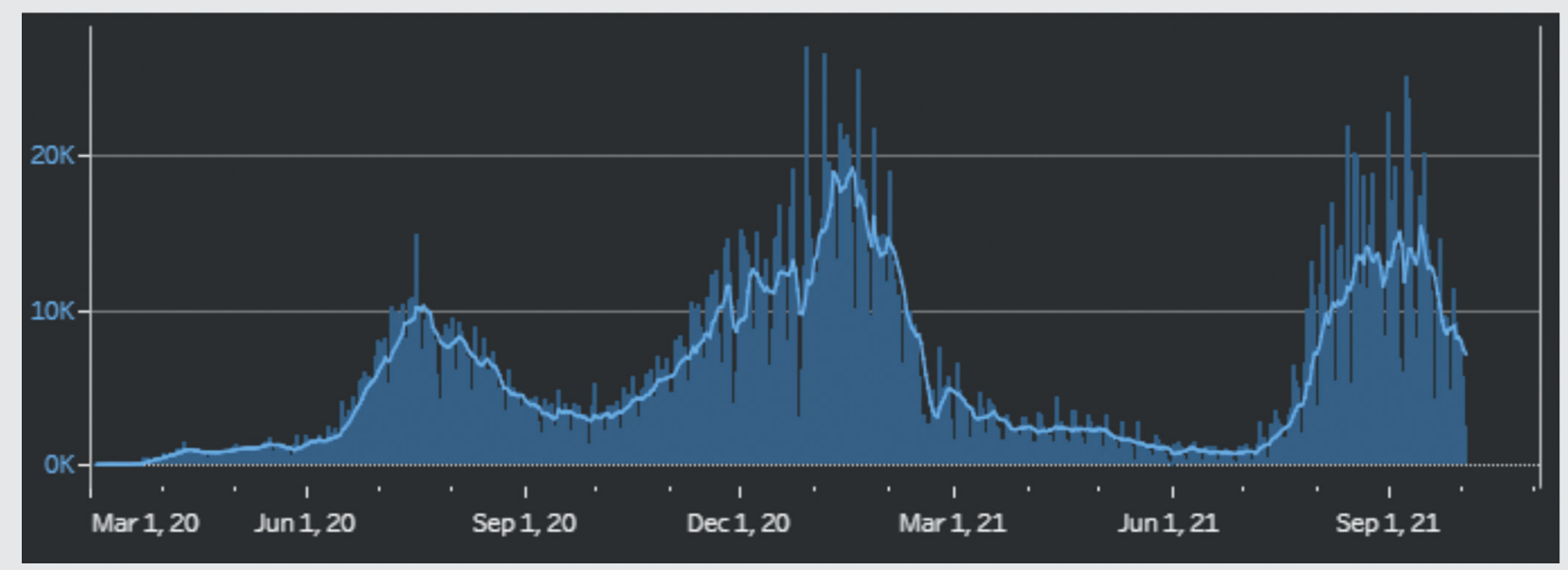

Figure 3. Daily new cases attributed to COVID-19 in the state of Texas. Bars are shown for each daily report. The curve is the 7-day moving average. Data are from the Texas Department of State Health Services. $^{2}$ 


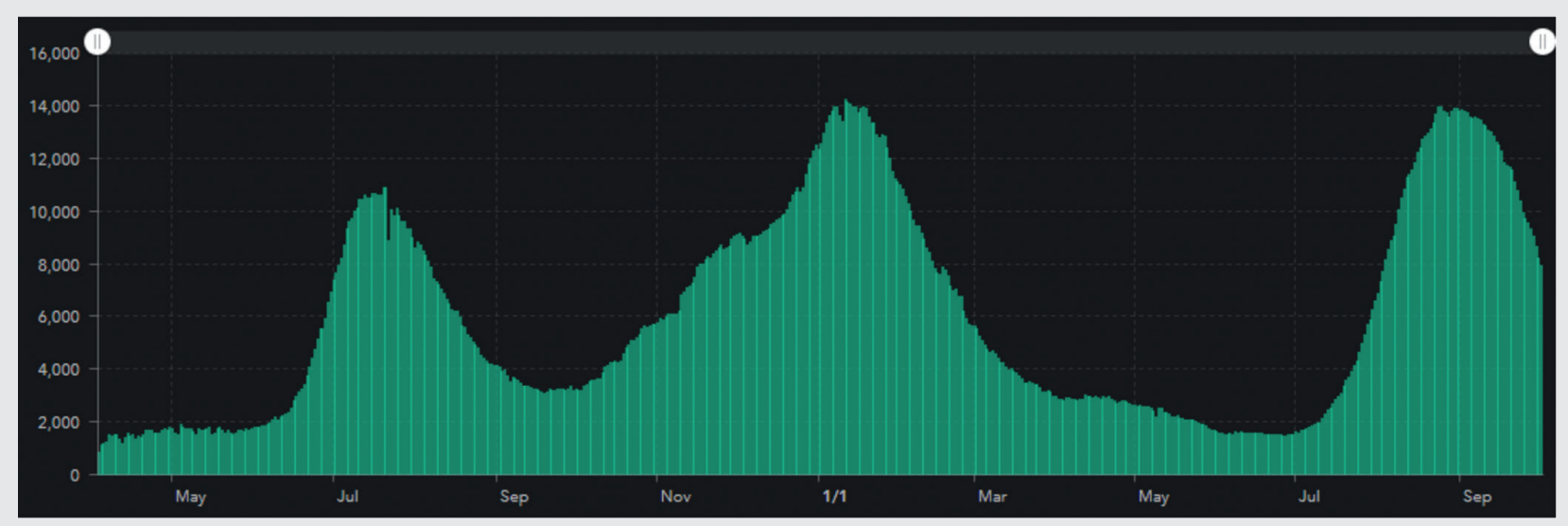

Figure 4. Daily Texas hospital beds occupied by patients with COVID-19. Data are from the Texas Department of Health Services. ${ }^{3}$

Figure 6 illustrates the new cases in Lubbock County. The data from Lubbock County track the data from Texas closely. There are substantial differences between the data from Texas and Lubbock County than from the United States. This demonstrates that individual regions may have much different patterns. For example, New York and New England had a much bigger problem in March and April of 2020 than Texas, but New York and New England had very little problem during the summer of 2020.
Figure 7 illustrates the hospitalizations in Texas Trauma Service Area B, which is mostly hospitals in Lubbock County. The data for 2021 are overlaid with the data from 2020 and illustrate that the timing of outbreaks has changed. The surge due to the Delta variant began around July 2021 , and the peak in hospitalizations occurred around September 7, 2021. Like the other figures, hospitalizations in Lubbock County are clearly in decline. This is very good news for the overworked intensivists caring for the most seriously ill patients with COVID-19.

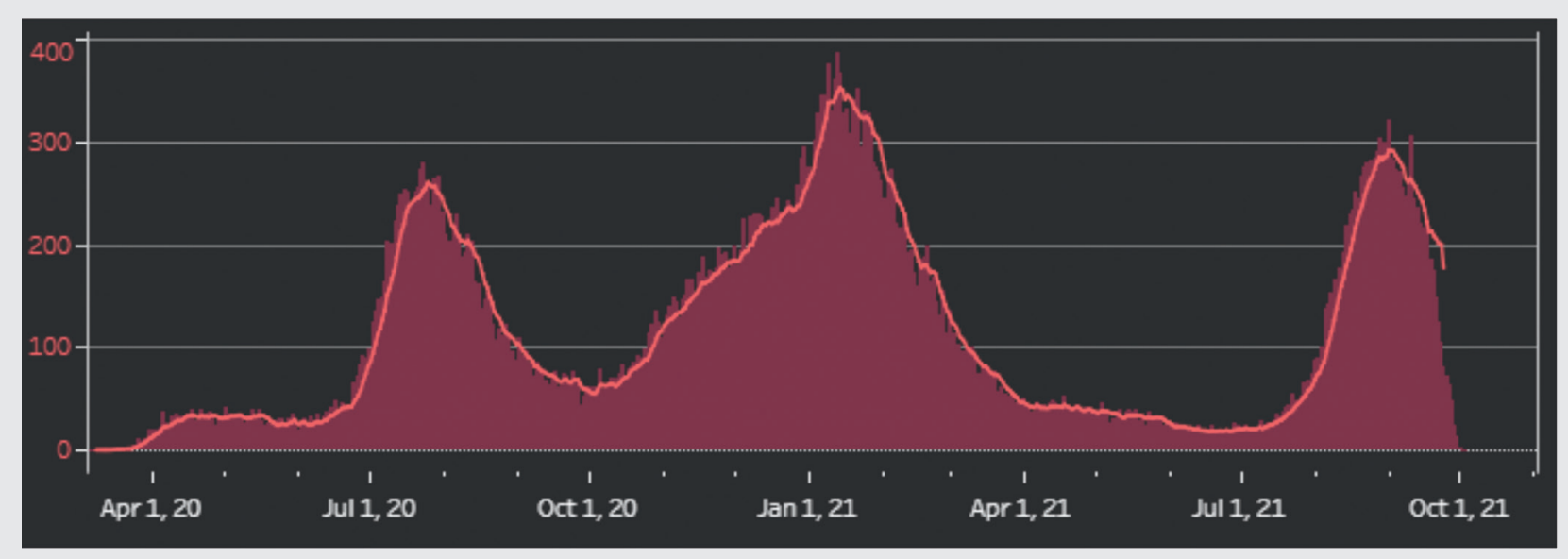

Figure 5. Daily deaths in Texas attributed to COVID-19. Data are from the Texas Department of State Health Services. ${ }^{2}$ 


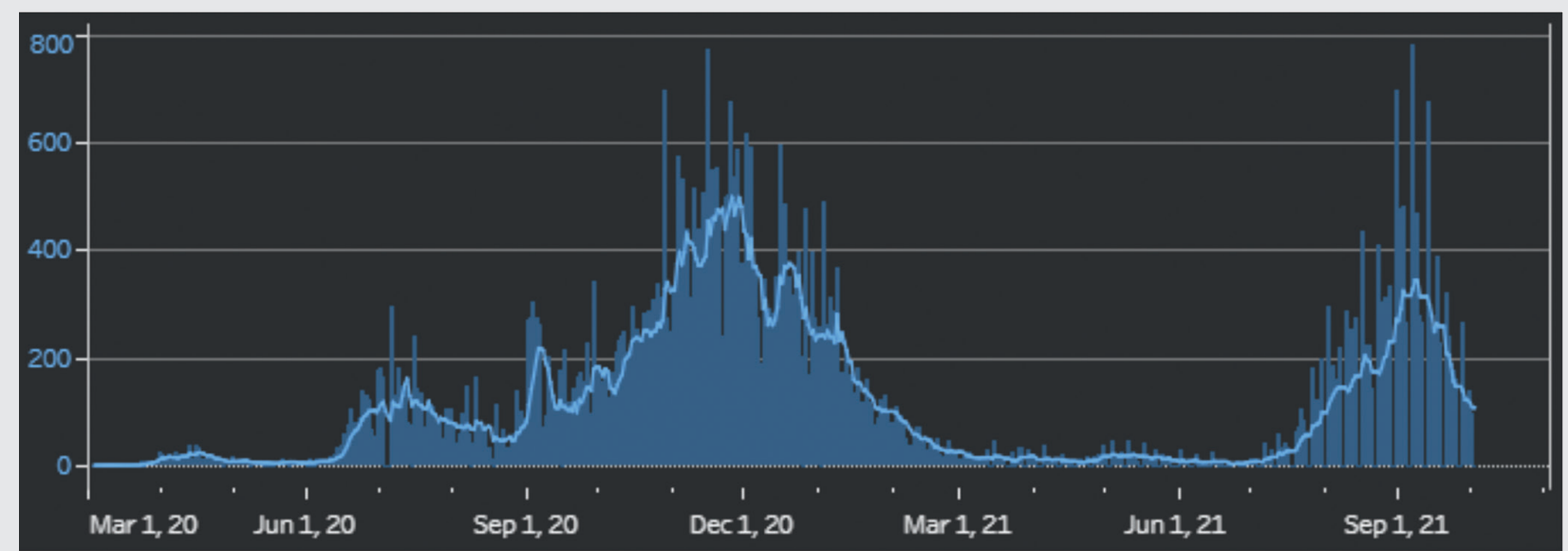

Figure 6. Daily new cases in Lubbock County attribute to COVID-19. Data are from the Texas Department of State Health Services. ${ }^{2}$ The surge due to the Delta variant began around July 1, 2021, and the peak in daily deaths occurred on September 9, 2021.

The figure illustrating deaths in Lubbock County is not shown as it tracks the Texas figure very closely. The peak in deaths in Lubbock County occurred on September 3, 2021, which is very close to the date of peak deaths in Texas.

The Delta variant is clearly in decline. However, there will eventually be a new outbreak with a new variant. Like influenza, the virus will always be ahead of the latest vaccines via mutations. The biggest mistake following the previous peak was to relax and assume that the problem was solved. Hopefully, we will learn from the previous mistakes so as not to repeat them.

During this outbreak, our hospital (University Medical Center in Lubbock, TX) was ill prepared for

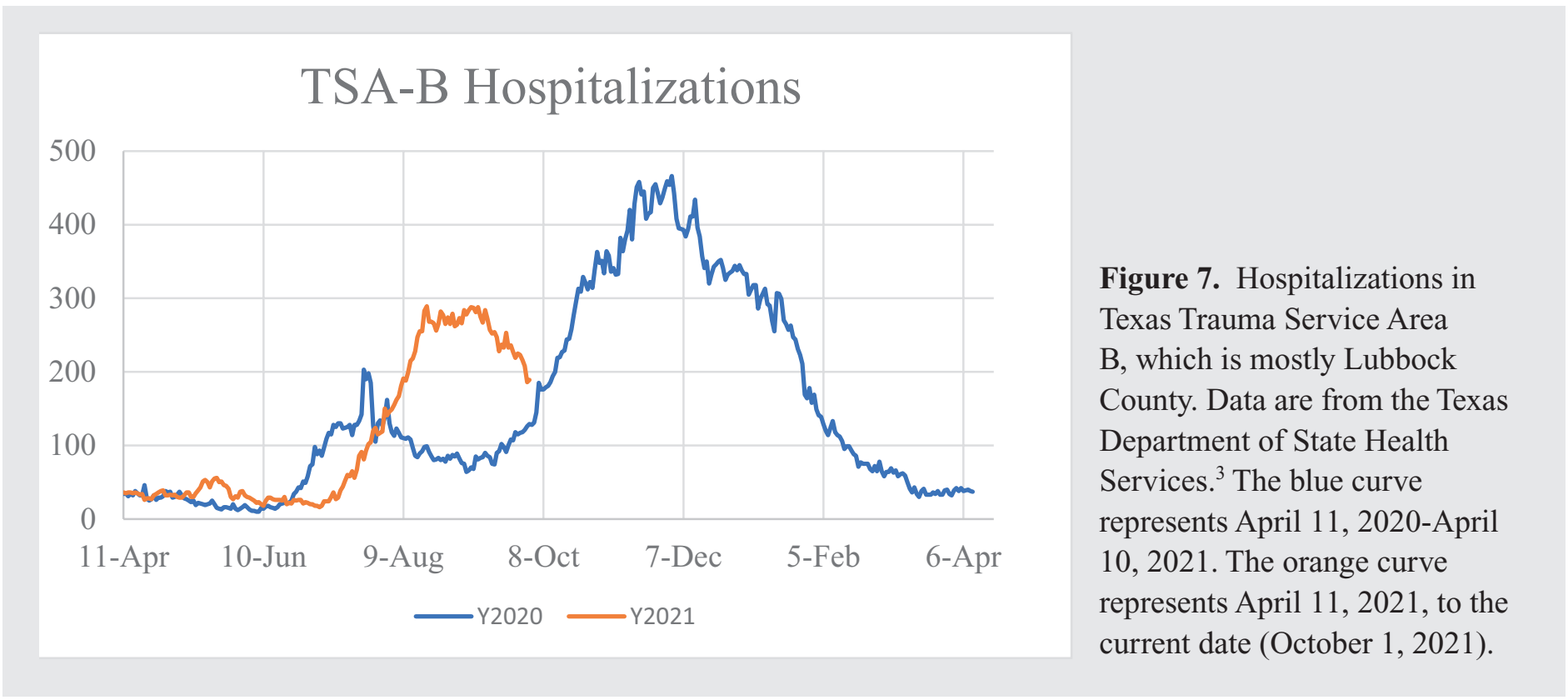


Delta. Many of the providers who pitched in to care for the patients on ventilators declined to do so for the Delta variant. The task of managing ventilator cases due to COVID-19 fell to the Pulmonary and Critical Care Medicine division, which had inadequate faculty, fellows, and residents to optimally manage the large number of patients across 4 different intensive care units on different floors in the medical center. Furthermore, other providers assumed that the usual level of service for procedures and pulmonary care outside of the intensive care units would continue. At the peak of this outbreak, one pulmonary fellow and one faculty physician were managing 20 COVID patients in the cardiac intensive care unit. Furthermore, two pulmonary fellows and one faculty physician were managing 32 patients in the medical intensive care unit. Although this welcome decline in COVID-19 will lead to normalization of workloads, it would be a mistake to assume that the recent past will not be repeated in terms of ventilator cases spread across 4 intensive care units. The hospital and the medical school will need a concrete plan on how to equitably distribute the workload during the inevitable next time when all 4 intensive care units are filled with COVID cases. deaths
Article citation: Berdine G. Delta variant is in decline in the US, Texas, and Lubbock County. The Southwest Respiratory and Critical Care Chronicles 2021;9(41):71-75 From: Department of Internal Medicine, Texas Tech University Health Sciences Center, Lubbock, Texas

Submitted: $10 / 4 / 2021$

Accepted: 10/6/2021

Conflicts of interest: none

This work is licensed under a Creative Commons Attribution-ShareAlike 4.0 International License.

\section{REFERENCES}

1. United States COVID. Worldometer. https://www.worldometers.info/coronavirus/country/us/ Accessed 10/4/2021.

2. Workbook: COVID County Trends Over Time. Texas Department of State Health Services. https://tabexternal.dshs. texas.gov/t/THD/views/COVIDCountyTrendsOverTime/ COVIDTrends?:isGuestRedirectFrom Vizportal $=y \&$ : embed $=\mathrm{y}$. Accessed 10/4/2021.

3. COVID-19 in Texas-Texas Tests and Hospitals. Texas Department of State Health Services. https://txdshs.maps. arcgis.com/apps/dashboards/0d8bdf9be927459d9cb11b9eaef6101f Accessed 10/4/2021. 